\title{
Vascular endothelial growth factor and KIT expression in relation with microvascular density and tumor grade in supratentorial astrocytic tumors ${ }^{1}$
}

\author{
Thaís Heinke', Kleber Simões do Espiríto Santo ${ }^{I I}$, Adhemar Longatto FilhoIII, João Norberto Stavale ${ }^{\mathrm{IV}}$
}

${ }^{\mathrm{I}} \mathrm{PhD}$, Associate Professor, Department of Pathology, Investigative Pathology Division, EPM-UNIFESP, Sao Paulo-SP, Brazil. Conception and design of the study, acquisition and interpretation of data, statistical analysis, drafting the article, revising and approving the final version.

${ }^{\text {II }} \mathrm{PDD}$, Clinical Hospital Luzia de Pinho Melo, Mogi das Cruzes-SP, Brazil. Interpretation of data, statistical analysis, revising and approving the final version.

IIIPhD, Laboratory of Medical Investigation (LIM) 14, Faculty of Medicine, University of Sao Paulo, Brazil. Interpretation of data, drafting the article, revising and approving the final version.

${ }^{\text {IV }} \mathrm{PhD}$, Associate Professor, Department of Pathology, Investigative Pathology Division, EPM-UNIFESP, Sao Paulo-SP, Brazil. Conception and design of the study, acquisition and interpretation of data, revising and approving the final version.

\section{ABSTRACT}

PURPOSE: To evaluate the relationship between microvascular density and the expression of vascular endothelial growth factor (VEGF) and KIT as possible markers of angiogenic stimulus in astrocytic tumors and correlate it with histopathological grading.

METHODS: We enrolled 99 surgical specimens of supratentorial astrocytic tumors for analysis of VEGF and KIT and subsequent correlation with MVD and grading.

RESULTS: KIT and VEGF expression correlated with microvascular density $(p<0.005)$ and both VEGF and microvascular density correlated with grading $(\mathrm{p}<0.005)$. KIT had no significant relationship with grading $(\mathrm{p}=0.657)$.

CONCLUSION: KIT and VEGF constitute important pathways in the angiogenesis of astrocytomas and therefore are promising prognostic tools and options for therapeutic intervention.

Key words: Glioma. Neovascularization, Pathologic. Immunohistochemistry. 
Vascular endothelial growth factor and KIT expression in relation with microvascular density and tumor grade in supratentorial astrocytic tumors

\section{Introduction}

Gliomas are a heterogeneous group of primary tumors in the central nervous system, and comprise 40 to $50 \%$ of all brain tumors. The most common type of gliomas are astrocytic tumors, a heterogeneous group of tumors, classified by degree of malignancy according to World Health Organization (WHO) criteria as diffuse astrocytomas (grade II), anaplastic astrocytomas (grade III) or glioblastomas (grade IV) ${ }^{1}$. Although glioblastomas may arise de novo, strong evidence indicates that some glioblastomas likely develop from low-grade astrocytomas; these tumors may also follow varying pathways of biological development ${ }^{2}$.

All of these histopathological types can recur, and can also be phenotypically malignant, depending on the location, patient's age, health status and the extent of surgical resection. Though astrocytomas course of development can vary, the resulting outcome is frequently dismal, despite current therapeutic strategies. Astrocytomas infiltrate the brain diffusely, spreading throughout adjacent healthy tissues and recurring even after radical surgical resection ${ }^{3}$.

Microvessel proliferation is one of the most important criteria for diagnosing malignancy in diffusely infiltrating astrocytic tumors ${ }^{4}$. Characterizing the molecular pathways leading to glioma angiogenesis and progression has clinical relevance because blocking molecular pathways that drive angiogenesis has major therapeutic implications ${ }^{5}$. Because high-grade gliomas are highly vascularized tumors, targeting blood vessels with antiangiogenic agents seems to be a promising treatment pathway ${ }^{6}$.

Although the factors controlling glioma angiogenesis are not fully understood, experimental data support the concept that angiogenesis is required for glioma growth and that the process is driven primarily by tumor-secreted vascular endothelial growth factor (VEGF) $)^{7,8}$. The formation of primitive blood vessels from progenitors like hemangioblasts and angioblasts is dependent on VEGF, which appears to play a major role in the angiogenesis of several other human tumors, such as colon and breast carcinomas.

The majority of anti-angiogenic therapies already interfere with the VEGF pathway, and the results are even more encouraging when anti-VEGF antibodies are combined with chemotherapy. Trials targeting the VEGF-signaling pathways are currently under development ${ }^{6}$.

Besides VEGF, tyrosine kinase receptors, such as KIT, are also being investigated as having an important role in the cytogenetic alterations of angiogenesis process $^{8}$. It is known that KIT, commonly present in juvenile brain tumors, also participate in glioma angiogenesis
The proto-oncogene KIT encodes CD117, a transmembrane tyrosine kinase that has a well-established role in several human tumors; cellular expression of CD117 has already been detected by immunohistochemistry in gliomas ${ }^{10}$. In addition to protein expression, it has also been suggested that KIT gene amplification may be observed more commonly in glioblastomas and astrocytic tumors than in oligodendrogliomas, which constitute gliomas outside the astrocytic lineage ${ }^{11}$. Therefore, the KIT amplification seems to be linked to the astrocytic tumors. During angiogenesis, the KIT pathway as well as VEGF expression appears involved with astrocytoma growth, invasiveness and malignancy ${ }^{11,12}$.

The degree of tumor vascularization can be measured by means of the microvessel density (MVD), which serves as an independent prognostic marker of survival in low-grade fibrillary astrocytomas ${ }^{4,13}$. It has also been suggested that tumor angiogenesis and vascular density can be used as determinants of radiosensitization, of response to anti-angiogenesis drugs and as reliable prognostic markers ${ }^{13,14}$.

Because KIT and VEGF represent attractive possibilities for personalized therapy strategies, we sought to evaluate by immunohistochemistry the relationship between MVD, KIT and VEGF status and the correlation of these with histopathological grades in a large series of supratentorial astrocytic tumors.

\section{Methods}

This is a retrospective study based on archived slides and paraffin blocks with central nervous system tumors from the Pathology Department of the Federal University of Sao Paulo, Brazil. Local Ethics Committee approved this study (CEP 1114/07).

From 2005 to 2011, we systematically searched for all paraffin blocks of supratentorial astrocytomas that were archived before patients underwent radiotherapy. To be eligible for the study, tumors had to be identified as astrocytomas grades II, III or IV ${ }^{1}$.

The specimens had been fixed in 10\% buffered formalin and embedded in paraffin in preparation for light microscopy on hematoxilin \& eosin (H\&E) stained slides. Two experienced neuropathologists (TH, JNS) analyzed all H\&E stained slides independently and the immunohistochemistry reactions using histopathological criteria outlined by the WHO to validate the original diagnosis, always reaching consensus. 


\section{Immunohistochemical reactions}

Immunohistochemical analysis was performed using $4 \mu \mathrm{m}$ sections according to the standard streptavidin-biotin immunoperoxidase method. CD34 antigen, a transmembrane protein expressed on hematopoietic stem cells and the small vessel endothelium of various tissues, was selected to visualize the endothelial cells for MVD evaluation. The monoclonal antibody against CD34 Xm (QBEnd 10) from DAKO (Co Carpinteria, CA, USA), m7165, was used at a 1:100 dilution. The other monoclonal antibodies used included VEGF xM (C-1) from Santa Cruz (Santa Cruz, CA. USA), sc 7269, at a 1:100 dilution, and c-KIT/CD 117 xR DAKO (Co Carpinteria, CA, USA), A 4502, at a 1:100 dilution. Deparaffinized and rehydrated slides were subjected to heatinduced antigen retrieval for 15 minutes in a pressure cooker with $10 \mathrm{mM}$ citrate buffer ( $\mathrm{pH} \mathrm{6.0)}$ at $120^{\circ} \mathrm{C}$ for $\mathrm{CD} 34$, or immersed in $10 \mathrm{mM}$ citrate buffer ( $\mathrm{pH} 6.0$ ) during microwave heating at $700 \mathrm{w}$ for 15 minutes for VEGF and KIT.

\section{Evaluation of immunohistochemistry staining}

Semiquantitative scores for VEGF and KIT were applied as following. Positive immunoreactions were categorized from 0 to 3 according to the extent and intensity of staining: grade 0 for no staining; grade 1 for weak focal staining; grade 2 for strong focal staining; and grade 3 for strong widespread staining.

VEGF and KIT antigen expressions were evaluated in the cytoplasm of neoplastic astrocytes. This graduated scale was adapted from Abduldrauf et al. ${ }^{13}$. For statistical analysis purposes, 0 and 1 were classified as negative, and grades 2 and 3 were grouped as positive.

Immunostained slides for CD34 were scanned field per field at 20x to facilitate the identification of positive reactions among vascular "hot spots". Microvessel counting was performed using a 100x magnification in two different fields at the site previously defined as the "hot spots". Images of these two fields were captured with a Digital Camera System DinoCapture, and the microvessels were manually identified using the free image processing and analyzing program Image Tool 3.00. If a discrepancy occurred in the number of vessels between the assessments of the two observers, the images were reassessed for a consensual analysis. MVD was expressed, in this study, as the number of vessels counted in the two hotspots.

Slides stained for CD34 were scanned at 20x for the identification of positive reactions. Two fields considered representative of vascularity were designated as vascular "hot spots". Images of the two hot spots were captured with a Digital Camera System DinoCapture using a 100x magnification, and the microvessels were identified and counted using the free image processing and analyzing program Image Tool 3.00. If a discrepancy occurred in the number of vessels between the assessments of the two observers, the images were reassessed for a consensual analysis. MVD was expressed, in this study, as the number of vessels counted in the two fields named as hot spots.

\section{Statistical analysis}

Statistical calculations were performed using SPSS 13.0 (SPSS Inc; Chicago, IL). In the case of a $\mathrm{p}<0.05$, the result was considered statistically significant. Associations of MVD and KIT or VEGF expression were verified with the Mann-Whitney test; associations between MVD and tumor grade were analyzed with the Kruskal-Wallis test; and associations between tumor grade and KIT or VEGF expression were tested with Pearson chi-square tests (two-sided).

\section{Results}

The search retrieved material from 99 patients with supratentorial astrocytic tumors: 62 were men. Patients had a mean age of 46 years (range 20-78 years). Among the 99 patients, $58(58.6 \%)$ had diffuse astrocytomas (WHO grade II); 25 (25.3\%) had anaplastic astrocytomas (WHO grade III); and $16(16.2 \%)$ of the patients had glioblastomas (WHO grade IV).

VEGF expression in grades II and III astrocytomas and in glioblastomas is described in Table 1.

TABLE 1 - Mean microvascular density (MVD) and assessment of vascular endothelial growth factor (VEGF) and KIT positive immunohistochemical reactions distributed according to tumor grade.

\begin{tabular}{cccc}
\hline $\begin{array}{c}\text { Grade } \\
\text { by WHO }\end{array}$ & $\begin{array}{c}\text { MVD } \\
\text { (median; range) }\end{array}$ & $\begin{array}{c}\text { VEGF } \\
\text { positivity }\end{array}$ & $\begin{array}{c}\text { KIT } \\
\text { positivity }\end{array}$ \\
\hline II & $24(13-44)$ & $25(43.1 \%)$ & $15(25.9 \%)$ \\
\hline III & $33(22-38)$ & $16(64 \%)$ & $7(28 \%)$ \\
\hline IV & $35(28-49)$ & $12(75 \%)$ & $6(37.5 \%)$ \\
\hline Total & $29(13-49)$ & $53(53.5 \%)$ & $28(28.3 \%)$ \\
\hline
\end{tabular}

WHO=World Health Organization ${ }^{1}$

KIT expression was detailed in the same Table. In the vast majority of cases the expression for both markers, when 
present, was diffuse.

\section{Correlation of MVD with KIT and VEGF expression}

The MVD was significantly higher for the group of tumors that expressed VEGF $(\mathrm{p}<0.005)$. The median MVD of the positive group was 34 (range 21-49), whereas the negative-forVEGF group had a median of 23 (range 13-35), as illustrated in Figure 1.

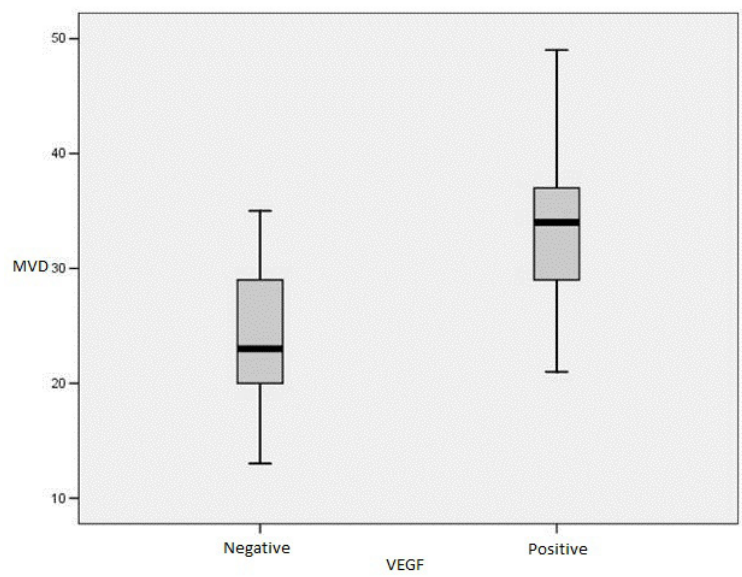

FIGURE 1 - Correlation between microvessel density (MVD) and vascular endothelial growth factor (VEGF) expression (Mann-Whitney test).

MVD was also significantly higher for the group of tumors that expressed KIT $(p<0.005)$. The median MVD of the positive group was 34 (range 27-49), whereas the negative for the KIT group had a median of 25 (range 13-39), as shown in Figure 2.

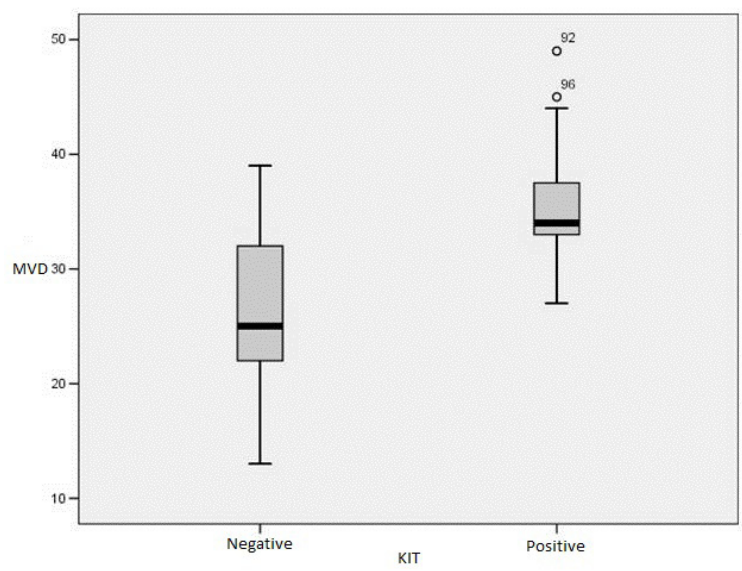

FIGURE 2 - Correlation between microvessel density (MVD) and KIT expression (Mann-Whitney test).

Tumor MVD was significantly associated with tumor grading $(\mathrm{p}<0.005)$, as shown in Figure 3 .

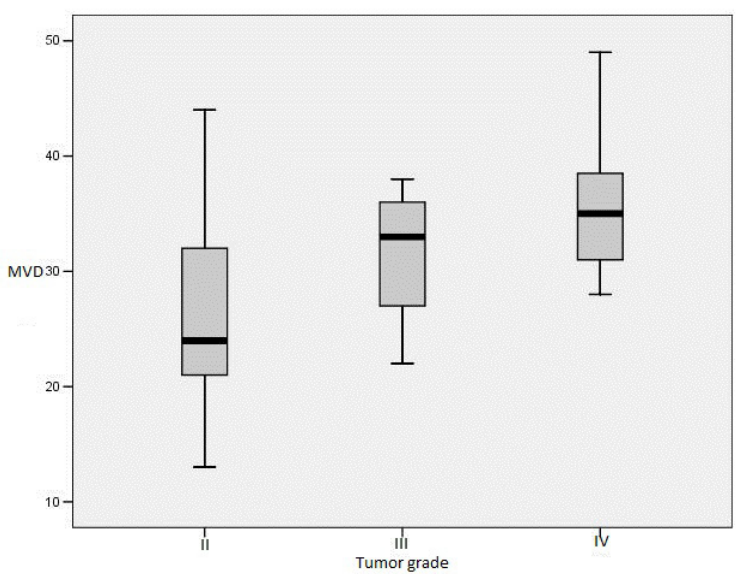

FIGURE 3 - Correlation between microvessel density (MVD) and tumor grade (Kruskal Wallis test).

Correlation of tumor grading and VEGF and KIT expression

The grading of gliomas correlated significantly with VEGF expression $(p<0.037)$. There was no association between KIT expression and tumor grade $(\mathrm{p}=0.657)$. Images of KIT and VEGF expression as well as microvascular density with CD34 staining are shown in Figure 4.

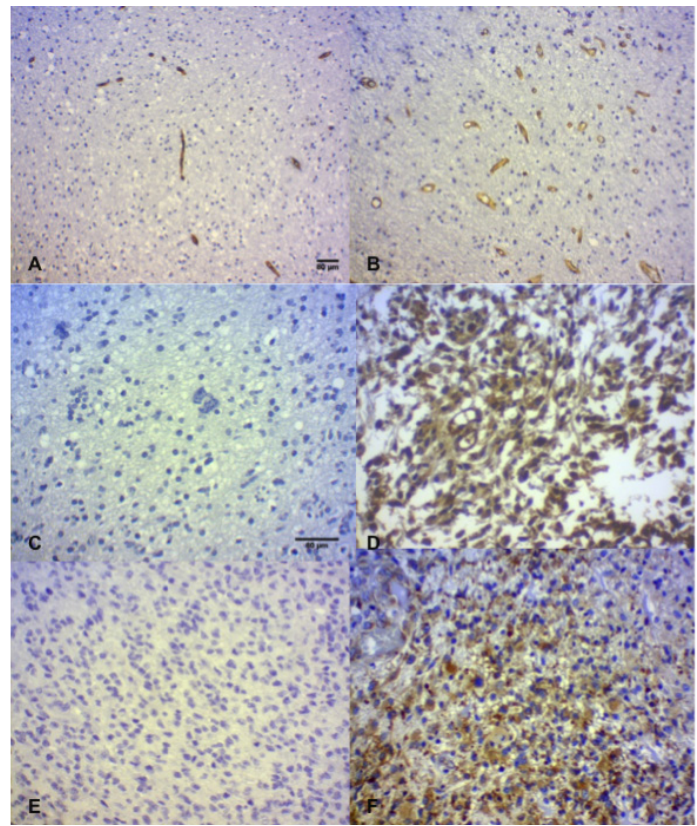

FIGURE 4 - A. Low microvessel counting in a diffuse astrocytoma (grade II). B. High microvessel counting in an anaplastic astrocytoma (grade III). C. Vascular endothelial growth factor (VEGF) expression absent in an anaplastic astrocytoma (grade III). D. VEGF expression positive in a glioblastoma (grade IV). E. KIT expression absent in an anaplastic astrocytoma (grade III). F. KIT expression positive in an anaplastic astrocytoma (grade III). (A, B: CD34 staining, original magnification 100x; C, D: VEGF staining, original magnification 200x; E, F: KIT staining, original magnification 200x). 


\section{Discussion}

Low-grade astrocytomas constitute a minor group of all astrocytic tumors and have an inherent tendency to become higher grade tumors through malignant transformation. Despite some well-known factors related to prognosis, no unambiguous marker can predict astrocytoma progression ${ }^{15}$. Astrocytomas are a heterogeneous group of gliomas, and accurate histopathological diagnosis is crucial to define the prognosis of these tumors, which differ primarily in their abilities to grow and progress ${ }^{1}$.

The malignant astrocytoma variants (grades III and IV) are the most frequently diagnosed primary brain tumors in adults. The migratory behavior of these malignant cells limits the effectiveness of surgical treatment, and the life expectancy of patients with glioblastomas, using current standard care, is an average of 14 months after diagnosis, despite the type of surgery, radiation and chemotherapies employed ${ }^{3}$.

Tumor microenvironments and genetic abnormalities that drive glioma growth, progression to malignancy and resistance to therapy are critical to optimize prognostic accuracy and to design optimal therapeutic strategies. Angiogenesis is fundamental for human reproduction and for wound healing, but it is also crucial for tumor progression, growth and metastasis. Angiogenesis is often present in high-grade lesions, and can be used as a prognostic factor. Many studies have focused on these characteristics and associations $^{16,17}$.

Leon et al. ${ }^{16}$ reported rich angiogenic activity in gliomas. Brain tumor growth was shown to depend largely on blood supply to provide nutrients and to remove necrotic debris from the central area of the neoplasm; a reciprocal relationship exists between the hemostatic system and tumor growth, cellular differentiation and metastasis. MVD is the final step of the whole process, and research dedicated to it allowed many authors to correlate certain tumors' biological aggressiveness with vascular proliferation ${ }^{17}$. In our study, we found a strong relationship between vascular proliferation and tumor grade $(\mathrm{p}<0.005)$, which reinforces the importance of MVD status and glioma classification and prognosis because malignant astrocytoma progression is heralded and accompanied by increased angiogenesis ${ }^{18}$.

Usually, molecular control of the angiogenic process entails regulating multiple steps that involve the recruitment and proliferation of endothelial cells, mediated by proangiogenic factors. Although there are many known angiogenic factors, vascular endothelial growth factor (VEGF) is certainly the major regulator in the case of gliomas and many other tumors ${ }^{13}$. Some reports have described the positive correlation of VEGF, vascular density and perfusion-weighted-imaging in gliomas ${ }^{19}$. The findings reported herein support this correlation because we found a relevant positive relation between VEGF expression and MVD $(\mathrm{p}<0.005)$. VEGF expression was also found to be significantly related to tumor grade $(\mathrm{p}=0.037)$. Yoo et $a .^{20}$ reported similar findings in a series of $78 \mathrm{WHO}$ grade II, III and IV astrocytic tumors. The cytokine VEGF is a multifunctional, endothelial cellspecific mitogen and angiogenesis inducer in vivo, and, currently, a number of clinical trials targeting the VEGF-signaling pathways are being developed ${ }^{6}$. Bevacizumab, a humanized anti-VEGF antibody has shown promising results in exploratory phase II trials of recurrent $\mathrm{GBM}^{21}$. Unfortunately, the response to anti-VEGF therapy is usually transient, and the majority of patients eventually relapsed, so new data is needed to understand the mechanisms of tumor escape ${ }^{22}$.

Another interesting therapeutic option is the novel targeted agent for KIT. Stem cell factor (SCF) is the ligand of the KIT receptor tyrosine kinase, which is codified by the gene KIT, located on chromosome 4q12 along with two other receptors named PDGFRA and VEGFR2, all of which are frequently amplified in primary glioblastomas ${ }^{12}$. SCF is up-regulated in highgrade gliomas; it promotes angiogenesis and the survival of central nervous cells and is associated with a short survival ${ }^{23}$. Because those receptors are preferentially expressed in tumor cells, their inhibition poses an attractive therapeutic option that might result in far fewer side effects than that observed in standard treatments.

$\mathrm{SCF} / \mathrm{KIT}$ signaling pathways are involved with nervous system development, and interact with signal transduction pathways involved in carcinogenesis and cellular differentiation that regulate many crucial steps of tumorigenesis; their activation is involved with angiogenesis and gametogenesis ${ }^{24}$. Stimulation of KIT with SCF results in dimerization, autophsphorylation and the resultant activation of downstream effector proteins that have already been widely investigated by neuroscientists because the KIT gene is highly amplified in both low and high-grade gliomas ${ }^{25}$. Primary human gliomas express KIT and induce normal neurons to express KIT in brain regions infiltrated by glioma cells when these areas present prominent angiogenesis ${ }^{26}$.

Sun and co-workers validated the hypothesis that KIT activation plays a fundamental role in glioma angiogenesis ${ }^{27}$. Its activation may promote these tumors' peculiar angiogenic patterns, which relate to gliomas' histopathological subtypes and vascular proliferation $^{28}$. Our findings support this hypothesis because we found a positive correlation between KIT expression and MVD $(p<0.005)$. Nevertheless, no correlation was found between the expression for KIT and the tumor grade $(\mathrm{p}=0.657)$. These findings 
are consistent with those of Gomes et al. ${ }^{11}$, in that out of a series of 179 gliomas of various grades, a positive expression for KIT was found in 25\% (1/4) of pilocytic astrocytomas, 25\% (5/20) of diffuse astrocytomas, $20 \%(1 / 5)$ of anaplastic astrocytomas and $19.5 \%$ $(15 / 77)$ of glioblastomas and no association was found between KIT tumoral overexpression and patient survival. Saini et al. ${ }^{29}$ also found that, contrary to earlier reports, KIT expression was seen in $32.5 \%$ (13/40) glioma cases with no association to tumor grade. Those findings of KIT over-expression across low/high grades in glioma cases are similar to ours and suggest that it may not be a molecular abnormality accumulated during tumor progression. Therefore, it is essential to elucidate whether the enhanced KIT expression is a cause or outcome of the glial neoplastic process ${ }^{29}$. Conversely, conflicting reports suggest that tumor aggressiveness depends on an increased expression of KIT and its activated form ${ }^{24}$. Cetin et al. ${ }^{10}$ studied a series of 52 glial tumors and found that $75 \%$ were positive for KIT, and the proportion of high-grade tumors was significantly greater than of low-grade tumors.

\section{Conclusion}

The vascular endothelial growth factor and KIT are markers associated with microvessel density and both VEGF and MVD correlated with grading. KIT had no significant relationship with grading in astrocytic tumors.

\section{References}

1. Brat DJ, Parisi JE, Kleinschmidt-DeMasters BK, Yachnis AT, Montine TJ, Boyer PJ, Powell SZ, Prayson RA, McLendon RE; Neuropathology Committee, College of American Pathologists. Surgical neuropathology update: a review of changes introduced by the WHO classification of tumours of the central nervous system, 4th edition. Arch Pathol Lab Med. 2008;132(6):993-1007.

2. Jaeckle KA, Decker PA, Ballman KV, Flynn PJ, Giannini C, Scheithauer BW, Jenkins RB, Buckner JC. Transformation of low grade glioma and correlation with outcome: an NCCTG database analysis. J Neurooncol. 2011;104(1):253-9.

3. Van Meir EG, Hadjipanayis CG, Norden AD, Shu HK, Wen PY, Olson JJ. Exciting new advances in neuro-oncology: the avenue to a cure for malignant glioma. CA Cancer J Clin. 2010;60(3):166-93.

4. Mahzouni P, Mohammadizadeh F, Mougouei K, Moghaddam NA, Chehrei A, Mesbah A. Determining the relationship between "microvessel density" and different grades of astrocytoma based on immunohistochemistry for "factor VIII-related antigen" (von Willebrand factor) expression in tumor microvessels. Indian $\mathrm{J}$ Pathol Microbiol. 2010;53(4):605-10.

5. Morris PG, Abrey LE. Novel targeted agents for platelet-derived growth factor receptor and c-KIT in malignant gliomas. Target Oncol. 2010;5(3):193-200.

6. Miletic H, Niclou SP, Johansson M, Bjerkvig R. Anti-VEGF therapies for malignant glioma: treatment effects and escape mechanisms. Expert Opin Ther Targets. 2009;13(4):455-68.
7. Hlobilkova A, Ehrmann J, Knizetova P, Krejci V, Kalita O, Kolar Z. Analysis of VEGF, Flt-1, Flk-1, nestin and MMP-9 in relation to astrocytoma pathogenesis and progression. Neoplasma. 2009;56(4):284-90.

8. Ambroise MM, Khosla C, Ghosh M, Mallikarjuna VS, Annapurneswari S. The role of immunohistochemistry in predicting behavior of astrocytic tumours. Asian Pac J Cancer Prev. 2010;11(4):1079-84.

9. Shibuya M. Brain angiogenesis in developmental and pathological processes: therapeutic aspects of vascular endothelial growth factor. FEBS J. 2009;276(17):4636-43.

10. Cetin N, Dienel G, Gokden M. CD117 expression in glial tumors. J Neurooncol. 2005;75(2):195-202.

11. Gomes AL, Reis-Filho JS, Lopes JM, Martinho O, Lambros MB, Martins A, Schmitt F, Pardal F, Reis RM. Molecular alterations of KIT oncogene in gliomas. Cell Oncol. 2007;29(5):399-408.

12. Sihto H, Sarlomo-Rikala M, Tynninen O, Tanner M, Andersson LC, Franssila K, Nupponen NN, Joensuu H. KIT and platelet-derived growth factor receptor alpha tyrosine kinase gene mutations and KIT amplifications in human solid tumors. J Clin Oncol. 2005;23(1):4957.

13. Abdulrauf SI, Edvardsen K, Ho KL, Yang XY, Rock JP, Rosenblum ML. Vascular endothelial growth factor expression and vascular density as prognostic markers of survival in patients with low-grade astrocytoma. J Neurosurg. 1998;88(3):513-20.

14. Norden AD, Drappatz J, Wen PY. Novel anti-angiogenic therapies for malignant gliomas. Lancet Neurol. 2008;7(12):1152-60.

15. Ranjan M, Santosh V, Tandon A, Anandh B, Sampath S, Devi BI, Chandramouli BA. Factors predicting progression of low-grade diffusely infiltrating astrocytoma. Neurol India. 2011;59(2):248-53.

16. Leon SP, Folkerth RD, Black PM. Microvessel density is a prognostic indicator for patients with astroglial brain tumors. Cancer. 1996;77(2):362-72.

17. Dietrich J, Norden AD, Wen PY. Emerging antiangiogenic treatment for gliomas - efficacy and safety issues. Curr Opin Neurol. 2008;21(6):736-44.

18. Gupta K, Radotra BD, Banerjee AK, Nijhawan R. Quantitation of angiogenesis and its correlation with vascular endothelial growth factor expression in astrocytic tumors. Anal Quant Cytol Histol. 2004;26(4):223-9.

19. Maia AC Jr, Malheiros SM, da Rocha AJ, da Silva CJ, Gabbai AA, Ferraz FA, Stávale JN. MR cerebral blood volume maps correlated with vascular endothelial growth factor expression and tumor grade in nonenhancing gliomas. AJNR Am J Neuroradiol. 2005;26(4):77783.

20. Yoo H, Sohn S, Nam BH, Min HS, Jung E, Shin SH, Gwak HS, Lee SH. The expressions of carbonic anhydrase 9 and vascular endothelial growth factor in astrocytic tumors predict a poor prognosis. Int J Mol Med. 2010;26(1):3-9.

21. Huylebrouck M, Lv S, Duerinck J, Van Binst A, Salmon I, De Greve J, De Witte O, Luce S, Michotte A, D’Haens J, Neyns B. An observational study of the first experience with bevacizumab for the treatment of patients with recurrent high-grade glioma in two belgian university hospitals. J Oncol. 2012;2012:801306.

22. Gerstner ER, Batchelor TT. Antiangiogenic therapy for glioblastoma. Cancer J. 2012;18(1):45-50.

23. Sun L, Hui AM, Su Q, Vortmeyer A, Kotliarov Y, Pastorino S, Passaniti A, Menon J, Walling J, Bailey R, Rosenblum M, Mikkelsen T, Fine HA. Neuronal and glioma-derived stem cell factor induces angiogenesis within the brain. Cancer Cell. 2006;9(4):287-300.

24. Sihto H, Tynninen O, Bützow R, Saarialho-Kere U, Joensuu H. Endothelial cell KIT expression in human tumours. J Pathol. 2007;211(4):481-8. 
25. Pupputti M, Tynninen O, Sihto H, Blom T, Mäenpää H, Isola J, Paetau A, Joensuu H, Nupponen NN. Amplification of KIT, PDGFRA, VEGFR2, and EGFR in gliomas. Mol Cancer Res. 2006;4(12):927-34.

26. Skardelly M, Armbruster FP, Meixensberger J, Hilbig H. Expression of Zonulin, c-kit and Glial Fibrillary Acidic Protein in Human Gliomas. Transl Oncol. 2009;2(3):117-20.

27. Sun L, Hui AM, Pastorino S, Passaniti A, Menon J, Fine HA. Stem cell factor/c-Kit activation involved in glioma tumour angiogenesis. Proceedings of the American Association for Cancer Research. 2005(46):abstract 4659.

28. Healy EF, Johnson S, Hauser CR, King PJ. Tyrosine kinase inhibition: Ligand binding and conformational change in c-Kit and c-Abl. FEBS Lett. 2009;583(17):2899-906.

29. Saini M, Jha AN, Abrari A, Ali S. A subset of human gliomas shows over-expression of KIT without its amplification. Gene. 2012;497(2):155-63.

\section{Correspondence:}

Thaís Heinke

Escola Paulista de Medicina-UNIFESP

Departamento de Patologia - Edifício Lemos Torres

Rua Botucatu, 740

04023-900 São Paulo - SP Brasil

Tel.: (55 11)5576-4996 / 99538-6662

thais.heinke@ig.com.br

Received: August 21, 2012

Review: October 23, 2012

Accepted: November 22, 2012

Conflict of interest: none

Financial source: none

${ }^{1}$ Research performed at Department of Pathology, Investigative Pathology Division, Paulista School of Medicine (EPM), Federal University of Sao Paulo (UNIFESP), Brazil. Part of PhD degree thesis, Postgraduate Program in Anatomic Pathology and Clinical Pathology. Tutor: João Norberto Stavale. 